\title{
Review of: "Modelling COVID-19 dynamics and potential for herd immunity by vaccination in Austria, Luxembourg and Sweden"
}

\author{
Igor Pataro ${ }^{1}$ \\ 1 Universidad de Almería
}

Potential competing interests: The author(s) declared that no potential competing interests exist.

This work presents comprehensive research concerning ODE's model validation for representing the SARSCoV-2 pandemic evolution in Luxembourg, Austria, and Sweden. The authors proposed a model parameter identification procedure based on a fundamentals bibliography review. Then, the proposed model parameters were subsequently cross-validated using Bayesian inference, which could also provide its quantitative uncertainty. From a validated model, in which considers vaccinated, hospitalized, and undetected infected individuals, the authors investigate the future scenarios concerning herd immunity in the previously mentioned countries, evaluating the alternative forecasting with regard to social measures and vaccination campaigns variations. The achieved result is in consonance with most of the recent literature, that is, to control the COVID-19 pandemic, not only the vaccine is urgent, but keep the social distancing control is the primary vector to manage the virus spread, avoiding its mutation and keep the balance with the economic sector until the herd immunity is achieved.

I believe this work is properly structured, well written, presents clear concepts and brings critical reflections on future results. The authors investigated important model concepts and contributed crucial information about future developments, which is essential for today's decision-making.

I would appreciate it if the authors could have commented on the model's predictive capabilities. For example, the parameters change from wave to wave, and some vary significantly. In addition, the social interaction parameters can change every two weeks. So, I wonder how well and for how long the proposed model can predict the pandemic evolution keeping the last identified parameters fixed over the prediction horizon. It is clear that the authors have made optimistic and straightforward assumptions for future scenarios. Still, some comments about it could strengthen the model potential.

Furthermore, the piece-wise constant social interaction parameter is considered as a degree of freedom of the model. In other words, it is used as a model parameter for tuning and fitting its curves. Nevertheless, it would be closer to reality by presenting actual information about social mobility in the respective countries. Indeed, each country might use different data representations to display their social mobility difference during the pandemic, which could be challenging to standardize it for applying in a unique model for three different countries. Nevertheless, using real social mobility data and associating it to nonpharmaceutical government measures would increase the model representativeness and strength the 
methodology. 